\title{
Rapid, Cost-effective Peptide/nucleic acid-based Platform for Therapeutic Antibody Monitoring in Clinical Samples
}

Marco Mocenigo ${ }^{\mathrm{a}, \mathrm{b}, \#}$, Alessandro Porchetta ${ }^{\mathrm{c}, \#}$, Marianna Rossetti $^{\mathrm{c}}$, Erik Brass ${ }^{\mathrm{a}}$, Lucia Tonini ${ }^{\mathrm{a}}$, Luca Puzzia , Elda Tagliabued ${ }^{\mathrm{d}}$, Tiziana Triulzi ${ }^{\mathrm{d}}$, Bruna Marini ${ }^{\mathrm{a}}$, Francesco Riccic, ${ }^{\mathrm{*}}$ and Rudy Ippodrino ${ }^{\mathrm{a},}$,

a. Ulisse BioMed Labs, Area Science Park, SS 14, km 163.5 Trieste (Italy)

b. Molecular Genetics and Biotechnology PhD study programme, University of Nova Gorica, Vipavska 13, Nova Gorica, Slovenia

c. Department of Chemistry, University of Rome, Tor Vergata, Via della Ricerca Scientifica, Rome, Italy

d. Department of Research, Fondazione IRCCS Istituto Nazionale dei Tumori, Milan, Italy 


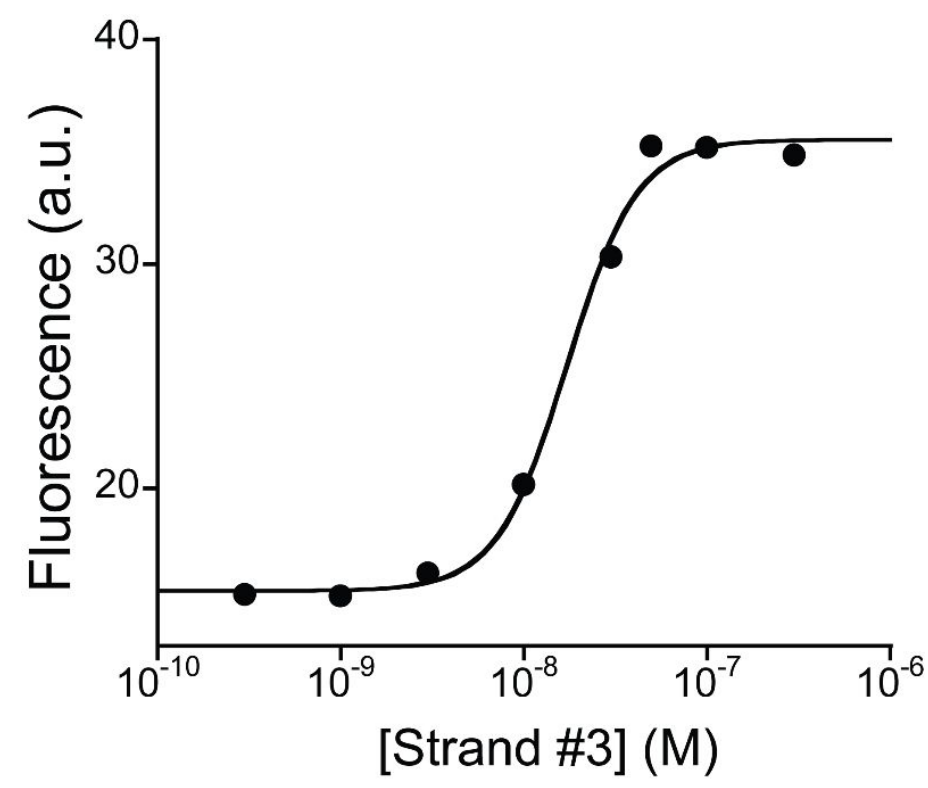

Figure SI1. Fluorescent binding assays obtained by adding increasing concentrations of PNA-peptide chimera probe (Strand \#3) to a fixed concentration of FAM/BHQ1 labelled DNA probe (Control DNA probe). This probe has been selected because its sequence is identical to the green portion of Strand \#1 and \#2 (10 nM) that is responsible for the binding with Strand \#3. The high affinity binding $(\mathrm{Kd}=20 \mathrm{nM})$ demonstrates the stoichiometric formation of the reporter (Strand $\#_{1}+$ Strand $\#_{3}$ ) and input modules (Strand \#2 + Strand \#3) at the experimental condition reported in the main text. Fluorescence experiments have been performed in $10 \%$ blood serum containing a fixed amount of control DNA probe (10 nM) at $25^{\circ} \mathrm{C}$. 


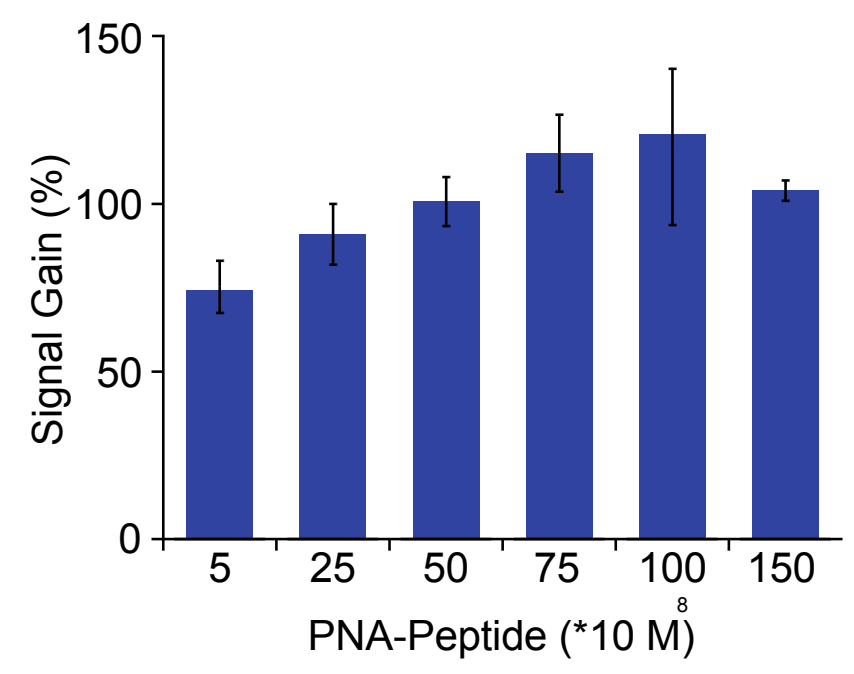

Figure SI2. Fluorescence signal gains of NanoHybrid in the presence of $200 \mathrm{nM}$ of Trastuzumab using different concentrations of PNA-peptide chimera probe (strand \#3). Fluorescence experiments have been performed in $10 \%$ blood serum containing the reporter module (strand \#1, 10 $\mathrm{nM}$ ) and the concentration of the PNA-peptide probe (strand $\#_{3}$ ) here reported by adding increasing concentrations of strand $\#_{2}$ in the presence $(200 \mathrm{nM})$ and absence of Trastuzumab at $25^{\circ} \mathrm{C}$. 


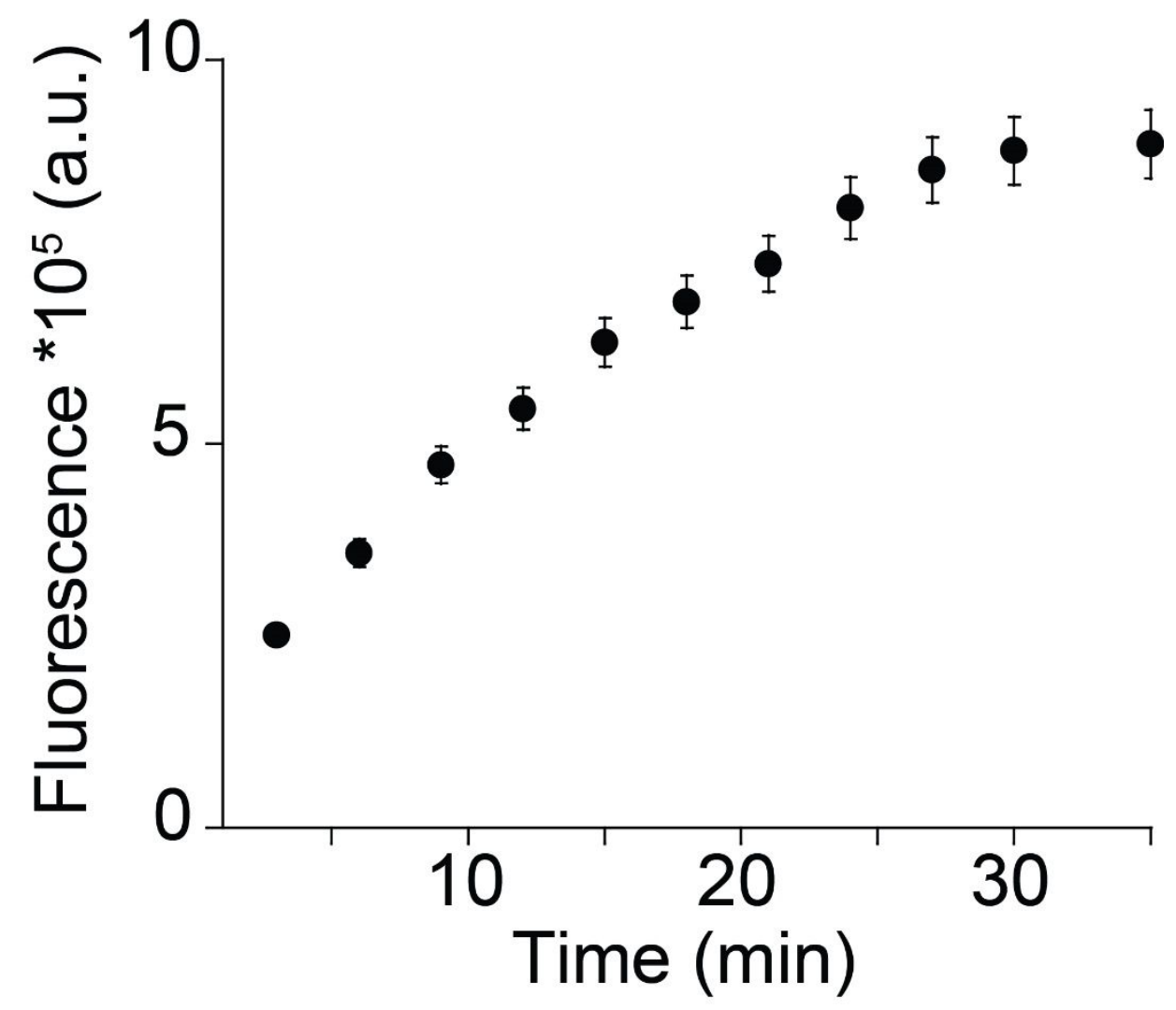

Figure SI3. Kinetics traces (fluorescence signal measured every 3 minutes) of NanoHybrid. Fluorescence experiments have been performed in $10 \%$ blood serum containing the reporter module (10 nM) and the input module (30 $\mathrm{nM})$ by adding Trastuzumab $(200 \mathrm{nM})$ at $25^{\circ} \mathrm{C}$. 